\title{
Linear and Nonlinear Electron Beam Heating of Magnetized, Motional, and Dusty Plasma
}

\author{
Sherif Mohamed Khalil'1,2, Badriah Mesfer AL Alotaibi1 \\ ${ }^{1}$ Physics Department, Faculty of Science, Princess Nora Bint Abdurrahman University, Riyadh, KSA \\ ${ }^{2}$ Plasma Physics \& Nuclear Fusion Department, N.R.C., Atomic Energy Authority, Cairo, Egypt \\ Email: smkhalil@pnu.edu.sa, mohamed_khalil@post.com
}

How to cite this paper: Khalil, Sh.M. and AL Alotaibi, B.M. (2016) Linear and Nonlinear Electron Beam Heating of Magnetized, Motional, and Dusty Plasma. Journal of Modern Physics, 7, 1889-1900.

http://dx.doi.org/10.4236/jmp.2016.714167

Received: September 1, 2016

Accepted: October 15, 2016

Published: October 19, 2016

Copyright $\odot 2016$ by authors and Scientific Research Publishing Inc. This work is licensed under the Creative Commons Attribution International License (CC BY 4.0).

http://creativecommons.org/licenses/by/4.0/

(c) (i) Open Access

\begin{abstract}
Recent theoretical work on electron beam heating of magneto-active motional Plas$\mathrm{ma}$ is presented. Power transfer from beam (plasma heating) and generated electric fields for different physical situations of linear and nonlinear beam-plasma interaction, are studied. Based on previous works [1] [2], we shall study the effects of dusts and plasma motion $\left(v_{o p} \neq 0\right)$ on plasma heating. Besides, the case of an inhomogeneity of beam velocity $\left(\frac{\partial v_{0 b}}{\partial x} \neq 0\right)$ is also considered. Taking into consideration nonlinear process, dust, plasma motion, and beam velocity inhomogeneity, are found to play a crucial role via power absorbed by the beam and the generated electric field in the system.
\end{abstract}

\section{Keywords}

Beam-Plasma Interaction, Motional Plasma, Dusty Plasmas, Plasma Heating, Electric Field Generation

\section{Introduction}

Electron beam-plasma interaction presents a great interest for many applications in areas like development of new methods in amplification and generation of electromagnetic waves, acceleration of charged particles in plasma (Plasma Accelerator). Besides, the electron beam-plasma system showed great importance via plasma generation, design of microwave tubes waveguides, explanation of natural phenomena that occur in space and solar plasmas, material studies, compact torus formation, generation of $\mathrm{x}$-ray and microwave, and others.

The recent development in the electron beam technology has shown the capability of generating powerful electron energy sources, making the electron beam very useful to 
controlled thermonuclear fusion research or in general, to plasma heating. Besides, the electron beam provides a free energy source for a rich variety of nonlinear process to evolve in a plasma. Accordingly, It is not surprising to find up till now a long list of studies and research on beam-plasma interaction and applications, which was also reviewed by many authors, (e.g., see [1]-[8] and references therein).

Multiple harmonic generation by laser/beam-plasma interaction has been widely investigated [9]-[13]. The generation of harmonics through a nonlinear mechanism driven by bunching at the fundamental has sparked interest as a path toward enhancing and extending the usefulness of an x-ray free-electron laser (FEL) facility. Besides, the sensitivity of nonlinear harmonic generation to electron beam quality is found to play a crucial role via FEL [14] [15].

Currently, high-order harmonic generation (HHG) is considered as one of the more efficient technique for producing coherent short-wavelength radiation in a broad spectral range [16]. Also, the nonlinear cold plasma-bunched beam interaction represents an interesting application for plasma wakefield accelerator [17].

From the point of view of beam-plasma interaction, the impact of dust on plasma is an important explosed field of research. It is increasingly being studied these days due to their applications in a wide range of fields. The presence of relatively highly charged and massive dust grains in a plasma can modify or influence the collective phenomena of the plasma. The possible dust modes may explain the extremely low-frequency fluctuations, new channels for the parametric coupling of other waves, generation of wakefields, etc., in dusty plasma. The impurities coming off from the walls of fusion device can create a dusty plasma at the edge of the discharge. These particles can enhance power loss due to radiation and dilution of fuel as well as can cool down the hot ion by charge-exchange process [18]-[23].

The subject of this paper is to study and investigate the electron beam heating of magneto-active motional dusty plasma under discuss different effects or parameters.

Section 2 explores the interaction of electron beam with a homogeneous magnetized non-motional plasma and plasma heating. Section 3 studies linear, non-motional $\left(v_{o p} \neq 0\right)$ and clean plasma in static magnetic field, while Section 4 is devoted to study the effects of the dust on the nonlinear beam interaction with non-motional plasma. Dusty plasma is characterized as a low-temperature ionized gas whose constituents are electrons, ions, and micron-sized dust particulates. The latter is usually negatively charged due to the attachment of the background plasma electrons on the surface of dust grains via collisions. The physical processes in dusty plasmas are interesting because of their importance for a number of applications in space plasmas and the earth $\mathrm{s}$ environment, as well as in the laboratory, and in several technologies. The dust grains have a strong effect on energy absorbed in the plasma. In Section 5, we study and investigates the effects of motional plasma $\left(v_{o p} \neq 0\right)$ on the heating processes due to beam-plasma interaction. Finally, in Section 6, we investigate the effects due to the inhomogeneity of beam velocity on both power absorption and generated electric field.

The electron beam is considered to be injected into plasma under the effect of static 
magnetic field $\boldsymbol{H}_{\text {ext }}=H_{o} \boldsymbol{e}_{\mathrm{z}}$. We use the well known expression for the amount of energy absorbed by the plasma per unit time $\mathrm{S}$ as:

$$
S=\frac{\omega}{2 \pi} \int \mathrm{d} x \operatorname{Im}|E|^{2}
$$

where $E$ is the electric field generated in the system.

\section{Linear, Non-Motional and Clean Plasma in Static Magnetic Field}

The equations of motion, and the continuity equation for electron beam, which travels along the magnetic field are:

$$
\begin{gathered}
\frac{\partial \boldsymbol{v}_{1 b}}{\partial t}+\boldsymbol{v}_{\circ b} \frac{\partial \boldsymbol{v}_{1 b}}{\partial x}=-\frac{e}{m}\left(\boldsymbol{E}+\frac{1}{c} \boldsymbol{v}_{1 b} \boldsymbol{H}_{\circ}\right) \\
\frac{\partial n_{1 b}}{\partial t}+v_{\circ b} \frac{\partial n_{1 b}}{\partial x}+n_{\circ b} \frac{\partial v_{1 b}}{\partial x}=0
\end{gathered}
$$

while the equation of motion, and the continuity equation for cold inhomogeneous plasma electrons in the static magnetic field are given by:

$$
\begin{gathered}
\frac{\partial \boldsymbol{v}_{1 p}}{\partial t}=-\frac{e}{m}\left(\boldsymbol{E}+\frac{1}{c} \boldsymbol{v}_{1 p} H_{0}\right) \\
\frac{\partial n_{1 p}}{\partial t}+n_{o p} \frac{\partial v_{1 p}}{\partial x}=0
\end{gathered}
$$

where, $N_{\alpha}=n_{o \alpha}+n_{1 \alpha}+n_{2 \alpha}+\cdots, V_{\alpha}=v_{o \alpha}+v_{1 \alpha}+v_{2 \alpha}+\cdots$ and $\alpha=(b ; p)$.

For non motional plasma $\boldsymbol{v}_{0 p}=0 . v_{0 b}, n_{0 b}$ and $\boldsymbol{v}_{0 p}, n_{0 p}$ are the unperturbed velocity and density of the beam and plasma respectively.

Solving the above system of equations we can derive the following expressions for the perturbed densities:

$$
\begin{gathered}
n_{1 b}=\frac{e n_{o b}}{m v_{o b}^{2}} \mathrm{e}^{i \frac{\omega}{v_{o b}} x} \int \mathrm{e}^{-i \frac{\omega}{v_{o b}} x}\left[E_{1}+\frac{i \omega}{v_{o b}} \mathrm{e}^{\frac{i \omega}{v_{o b}} x} \int E_{1}\left(x^{\prime}\right) \mathrm{e}^{\frac{-i \omega}{v_{o b}} x^{\prime}} \mathrm{d} x^{\prime}\right] \mathrm{d} x \\
n_{1 p}=\frac{-e}{m \omega \omega^{\prime}} \frac{\partial}{\partial x}\left(n_{1 p} E_{1}\right)
\end{gathered}
$$

where, $\omega^{\prime}=\omega+i \omega_{c}$, and $\omega_{c}$ is the cyclotron frequency.

Using relations (6) and (7) in Poisson's equation

$$
\frac{\partial E_{1}}{\partial x}=-4 \pi e\left(n_{1 p}+n_{1 b}\right)
$$

we obtain the following wave equation for the electric field:

$$
\left(v_{o b} \frac{\partial}{\partial x}-i \omega\right)^{2}\left(\varepsilon(x) \boldsymbol{E}_{1}(x)\right)+\omega_{b}^{2} \boldsymbol{E}_{1}(x)=\text { const }
$$

where, $\omega_{c}=\frac{e H_{O}}{m c}, \omega_{s}^{2}=\frac{4 \pi e^{2} n_{o s}}{m} ;(s=(b, p)), \varepsilon(x)=1-\frac{\omega_{p}^{2}}{\omega \omega^{\prime}}$

Introducing the electric field form: $\boldsymbol{E}(x)=\frac{F(x)}{\varepsilon(x)} \mathrm{e}^{\frac{i \omega}{v_{o b}} x}$, Equation (9) reduces to: 


$$
\frac{\mathrm{d}^{2} F}{\mathrm{~d} x}+\frac{\chi^{2}}{\varepsilon(x)} F(x)=0, \text { where, } \chi^{2}=\left(\frac{\omega_{b}}{\boldsymbol{v}_{{ }_{b}}}\right)^{2}
$$

Then we can write the final solution of (9) in the simple form:

$$
\boldsymbol{E}_{1} \cong \frac{F_{01}}{\varepsilon(x)} \mathrm{e}^{\frac{i \omega}{v_{o b}} x}, F_{01}=\text { const. }
$$

Set (11) into (1), the energy absorbed absorbed by the beam reads:

$$
S=\frac{F_{01} \omega}{2 \pi} \int \frac{1}{\varepsilon(x)} \mathrm{d} x=\frac{F_{01} \omega}{2 \pi} \int\left(1-\frac{\omega_{p}^{2}}{\omega \omega^{\prime}}\right)^{-1} \mathrm{~d} x
$$

Relation (12) is investigated for different cases of magnetic field: zero, weak and strong magnetic fields.

\section{Linear Dusty Plasma Heating}

For dusty plasma, we use the system of Equations (2)-(5) with $\alpha=d$, then we obtain the following equation of motion, and the continuity equation as:

$$
\begin{gathered}
\frac{\partial \boldsymbol{v}_{1 d}}{\partial t}+\boldsymbol{v}_{\circ_{d}} \frac{\partial \boldsymbol{v}_{1 d}}{\partial x}=-\frac{e z_{d} n_{d}}{m_{d} n_{d}} \boldsymbol{E} \\
\frac{\partial n_{1 d}}{\partial t}+\frac{\partial}{\partial x}\left(n_{d} v_{d}\right)=0
\end{gathered}
$$

Solving the above system of equations we can derive the following expressions for the dust perturbed density:

$$
n_{1 d}=\frac{-z_{d} e}{m_{d} \omega^{2}} \frac{\partial}{\partial x}\left(n_{d} E_{1}\right)
$$

where, $z_{d}$ Charge of dust.

Using relations (7) and (8), (15) in Poisson's equation:

$$
\frac{\partial E_{1}}{\partial x}=-4 \pi e\left(-z_{d} n_{1 d}+n_{1 p}+n_{1 b}\right)
$$

we obtain the following wave equation for the perturbed electric field:

$$
\left(v_{o b} \frac{\partial}{\partial x}-i \omega\right)^{2}\left(\varepsilon_{d}(x) \boldsymbol{E}_{1}(x)\right)+\omega_{b}^{2} \boldsymbol{E}_{1}(x)=\text { const }
$$

where, $\varepsilon(x)+\frac{\omega_{d}^{2}}{\omega^{2}}=\varepsilon(x)+\Delta \varepsilon(x), \quad \varepsilon_{d}(x)=1-\frac{\omega_{p}^{2}}{\omega \omega^{\prime}}+\frac{\omega_{d}^{2}}{\omega^{2}}$.

The energy absorbed in this case reads:

$$
S_{d}=\frac{F_{02} \omega}{2 \pi} \int \frac{1}{\varepsilon_{d}(x)} \mathrm{d} x=\frac{F_{02} \omega}{2 \pi} \int\left(1-\frac{\omega_{p}^{2}}{\omega \omega^{\prime}}+\frac{\omega_{d}^{2}}{\omega^{2}}\right)^{-1} \mathrm{~d} x, \quad F_{02}=\text { const }
$$

In linear regime, if $S_{c}$ and $S_{d}$ are the energy absorbed by the beam in clean and in dusty plasma, respectively, then: 


$$
\frac{S_{d}}{S_{c}}=1-\frac{\Delta S_{d}}{S_{c}}<1 ; \frac{\Delta S_{d}}{S_{c}}=\frac{\omega_{d}^{2}}{\omega^{2}}
$$

It is clear that, existence of dust leads to less energy absorption by the plasma.

The generated electric field under the effect of dusty and clean plasma is shown in Figure 3.

\section{Nonlinear Dusty Plasma Heating}

In nonlinear regime, the equation of motion, and the continuity equation for electron beam, which travels along the magnetic field are:

$$
\begin{gathered}
\frac{\partial \boldsymbol{v}_{2 b}}{\partial t}+\boldsymbol{v}_{1 b} \frac{\partial \boldsymbol{v}_{1 b}}{\partial x}+\boldsymbol{v}_{\circ b} \frac{\partial \boldsymbol{v}_{2 b}}{\partial x}=-\frac{e}{m}\left(\boldsymbol{E}+\frac{1}{c} \boldsymbol{v}_{2 b} H_{\circ}\right) \\
\frac{\partial n_{2 b}}{\partial t}+v_{o b} \frac{\partial n_{2 b}}{\partial x}+v_{1 b} \frac{\partial n_{1 b}}{\partial x}+n_{1 b} \frac{\partial v_{1 b}}{\partial x}=0
\end{gathered}
$$

The equation of motion, and the continuity equation for homogeneous plasma electrons in a static magnetic field perpendicular to the plasma are given by:

$$
\begin{gathered}
\frac{\partial \boldsymbol{v}_{2 p}}{\partial t}+\boldsymbol{v}_{1 p} \frac{\partial \boldsymbol{v}_{1 p}}{\partial x}=-\frac{e}{m}\left(\boldsymbol{E}+\frac{1}{c} \boldsymbol{v}_{2 p} \boldsymbol{H}_{\circ}\right) \\
\frac{\partial n_{2 p}}{\partial t}+v_{1 p} \frac{\partial n_{1 p}}{\partial x}+n_{1 p} \frac{\partial v_{1 p}}{\partial x}=0
\end{gathered}
$$

The dusty nonlinear equations of motion, and continuity are:

$$
\begin{gathered}
\frac{\partial \boldsymbol{v}_{2 d}}{\partial t}+\boldsymbol{v}_{1 d} \frac{\partial \boldsymbol{v}_{1 d}}{\partial x}+\boldsymbol{v}_{o d} \frac{\partial \boldsymbol{v}_{2 d}}{\partial x}=-\frac{e}{m}\left(\boldsymbol{E}+\frac{1}{c} \boldsymbol{v}_{2 d} \boldsymbol{H}_{o}\right) \\
\frac{\partial n_{2 d}}{\partial t}+v_{o d} \frac{\partial n_{2 d}}{\partial x}+v_{1 d} \frac{\partial n_{1 d}}{\partial x}+n_{1 d} \frac{\partial v_{1 d}}{\partial x}=0
\end{gathered}
$$

Solving the above system of equations we can derive the following expressions for the nonlinear perturbed densities:

$$
\begin{gathered}
n_{2 b}=\frac{-1}{v_{0 b}} \mathrm{e}^{\left(\frac{i \Omega}{v_{0 b}}\right) x} \int \frac{\partial}{\partial x}\left(n_{0 b} v_{2 b}\right) \mathrm{e}^{-\left(\frac{i \Omega}{v_{0 b}}\right) x} \mathrm{~d} x+J_{2 b}(x) \\
n_{2 p}=\frac{\partial}{\partial x}\left(\frac{e n_{0 p}}{m \Omega^{2}} E_{2}\right)+J_{2 p}(x) \\
n_{2 d}=\frac{\partial}{\partial x}\left(\frac{-Z_{d} e n_{d}}{m_{d} \Omega^{2}} E_{2}\right)+J_{2 d}(x)
\end{gathered}
$$

where, $\Omega=2 \omega$ (second harmonic generation), and

$$
\begin{gathered}
J_{2 b}(x)=\frac{-1}{v_{o b}} \mathrm{e}^{\frac{i \Omega}{v_{o b}} x} \int \mathrm{e}^{\frac{-i \Omega}{v_{o b}} x}\left(n_{1 b} \frac{\partial v_{1 b}}{\partial x}+v_{1 b} \frac{\partial n_{1 b}}{\partial x}\right) \\
J_{2 p}(x)=\frac{-i}{\Omega} \frac{\partial}{\partial x}\left(\frac{-i e^{2}}{\Omega \omega m^{2}} \boldsymbol{E}_{1} \cdot \frac{\partial \boldsymbol{E}_{1}}{\partial x}+\frac{i e^{2} n_{o p}}{m^{2} \omega^{3}}\left(\frac{\partial^{2}}{\partial x^{2}} E_{1}^{2}+E_{1} \frac{\partial E_{1}}{\partial x}\right)\right)
\end{gathered}
$$




$$
J_{2 d}(x)=\frac{-i}{\Omega} \frac{\partial}{\partial x}\left(\frac{-i e^{2}}{\Omega \omega m^{2}} \boldsymbol{E}_{1} \cdot \frac{\partial \boldsymbol{E}_{1}}{\partial x}+\frac{i e^{2} n_{d}}{m^{2} \omega^{3}}\left(\frac{\partial^{2}}{\partial x^{2}} E_{1}+E_{1} \frac{\partial E_{1}}{\partial x}\right)\right)
$$

Using relations (25)-(27), and considering the nonlinear form of Poisson's Equation (16), we obtain the following wave equation for the nonlinear generated electric field:

$$
\left(v_{o b} \frac{\partial}{\partial x}-i \omega\right)^{2}\left(\varepsilon_{2 d}(x) \boldsymbol{E}_{2}(x)\right)+\omega_{b}^{2} \boldsymbol{E}_{2}(x)=\text { const }
$$

where, $\varepsilon(x)+\frac{\omega_{d}^{2}}{\Omega^{2}}=\varepsilon(x)+\Delta \varepsilon(x), \quad \varepsilon_{2 d}(x)=1-\frac{\omega_{p}^{2}}{\Omega \omega^{\prime}}+\frac{\omega_{d}^{2}}{\Omega^{2}}$.

Accordingly, we obtain the energy absorption from the beam as:

$$
S_{2 d}=\frac{F_{03} \omega}{2 \pi} \int \frac{1}{\varepsilon_{2 d}(x)} \mathrm{d} x=\frac{F_{03} \omega}{2 \pi} \int\left(1-\frac{\omega_{p}^{2}}{\Omega \omega^{\prime}}+\frac{\omega_{d}^{2}}{\Omega^{2}}\right) \mathrm{d} x, \quad F_{03}=\text { const }
$$

From (18) and (29) we conclude that:

$$
\frac{S_{2 d}}{S_{d c}}=1-\frac{\Delta S_{2 d}}{S_{d}}<1
$$

Ratio (30) shows that, for nonlinear interaction the existence of dust leads to less energy absorption by the plasma as in the linear regime.

Figure 4 shows the generated electric field under the effect of dusty and clean nonlinear plasma. In this regime amplification of electric field in dusty plasma is clear compared to clean plasma.

Generally speaking, nonlinearity plays a crucial role in the amplification of the electric field generated due to beam-plasma interaction, for different situations, compared to linear case.

\section{Motional Plasma}

For motional plasma, for the electron beam, we use the above system of Equations (2), (3 and (6). On the other hand, the equation of motion, and the continuity equation for homogeneous plasma electrons in a static magnetic field perpendicular to the plasma are given by:

$$
\begin{gathered}
\boldsymbol{v}_{o p} \neq 0 \\
\frac{\partial \boldsymbol{v}_{1 p}}{\partial t}+\boldsymbol{v}_{o P} \frac{\partial \boldsymbol{v}_{1 p}}{\partial x}+\boldsymbol{v}_{1 p} \frac{\partial \boldsymbol{v}_{o p}}{\partial x}=\frac{-e}{m}\left(\boldsymbol{E}_{1}+\frac{1}{c} v_{o p} \boldsymbol{H}_{\circ}\right) \\
\frac{\partial n_{1 P}}{\partial t}+v_{o P} \frac{\partial n_{1 P}}{\partial x}+n_{o P} \frac{\partial v_{1 P}}{\partial x}=0
\end{gathered}
$$

Accordingly, we obtain the following expressions for the perturbed plasma density

$$
n_{1 p}=\frac{e n_{o p}}{m v_{o p}^{2}} \mathrm{e}^{i \frac{\omega}{v_{o p}} x} \int \mathrm{e}^{-i \frac{\omega}{v_{o p}} x}\left[\boldsymbol{E}_{1}+\frac{i \omega^{\prime}}{\boldsymbol{v}_{o p}} \mathrm{e}^{\frac{i \omega_{o p}^{\prime}}{v_{o p}}} \int \boldsymbol{E}_{1}\left(x^{\prime}\right) \mathrm{e}^{\frac{-i \omega^{\prime}}{\boldsymbol{v}_{o p}} x^{\prime}} \mathrm{d} x^{\prime}\right] \mathrm{d} x
$$

Using relations (6) and (34) in Poisson's Equation (8), we obtain the following wave equation for the electric field in motional plasma 


$$
\left(V \frac{\partial}{\partial x}-i \omega\right)^{2}\left(\varepsilon_{m o t .}(x) \boldsymbol{E}_{1}(x)\right)=0
$$

where,

$$
\varepsilon_{\text {mot. }}(x)=1-\left(\frac{\omega_{p}^{2}}{\omega^{2}}+\frac{v_{o b}^{2}}{V^{2}}\right), V^{2}=v_{o p}^{2}+v_{o b}^{2},
$$

In this case the energy absorbed is:

$$
S_{\text {mot. }}=\frac{F_{04} \omega}{2 \pi} \int \frac{1}{\varepsilon_{\text {mot. }}(x)} \mathrm{d} x=\frac{F_{04} \omega}{2 \pi} \int\left(1-\frac{\omega_{p}^{2}}{\omega^{2}}+\frac{v_{o b}^{2}}{V^{2}}\right) \mathrm{d} x
$$

From this relation and non-motional relation (12) we conclude that:

$$
\frac{S}{S_{\text {mot. }}}=\frac{\varepsilon_{\text {mot. }}(x)}{\varepsilon(x)}>1
$$

Ratio (37) shows that, the existence of plasma motion leads to less energy absorption from the beam, as indicated in Figure 6.

Figure 7 shows the reduction of the generated electric field under the effect of motional plasma compared to non-motional plasma.

\section{Inhomogeneity of Beam Velocity Effects}

Let us consider the case of an electron beam moves with inhomogeneous velocity, i.e., $\frac{\partial v_{0 b}}{\partial x} \neq 0$. In this case we have to include the term $v_{1 b} \frac{\partial \boldsymbol{v}_{0 b}}{\partial x}$ in both the equations of motion (2) and continuity. Accordingly, we obtain the perturbed beam velocity and density as:

$$
\begin{gathered}
\boldsymbol{v}_{1 b}=\frac{-e}{m \boldsymbol{v}_{0 b}} \mathrm{e}^{\left(\frac{i \omega^{\prime \prime}}{v_{0 b}}\right) x} \int \boldsymbol{E}_{1}(x) \mathrm{e}^{-\left(\frac{i \omega^{\prime \prime}}{v_{0 b}}\right) x} \mathrm{~d} x \\
n_{1 b}=\frac{e n_{0 b}}{m v_{0 b}^{2}} \mathrm{e}^{\left(\frac{i \omega}{v_{0 b}}\right) x} \int \mathrm{e}^{-\left(\frac{i \omega}{v_{0 b}}\right) x}\left[\boldsymbol{E}_{1}+\frac{i \omega^{\prime \prime}}{v_{0 b}} \mathrm{e}^{\left(\frac{i \omega^{\prime \prime}}{v_{0 b}}\right) x} \int \boldsymbol{E}_{1}\left(x^{\prime}\right) \mathrm{e}^{-\left(\frac{i \omega^{\prime \prime}}{v_{0 b}}\right) x^{\prime}} \mathrm{d} x^{\prime}\right] \mathrm{d} x
\end{gathered}
$$

where, $\omega^{\prime \prime}=\omega+\frac{\partial v_{0 b}}{\partial x}$, and the equation controlling the perturbed electric field is given by:

$$
\left(v_{o_{b}} \frac{\partial}{\partial x}-i \omega^{\prime \prime}\right)^{2}\left(\varepsilon(x) \boldsymbol{E}_{1}(x)\right)+\omega_{b}^{2} \boldsymbol{E}_{1}(x)=\text { const. }, \quad \varepsilon(x)=1-\frac{\omega_{p}^{2}}{\omega^{2}}
$$

Using mathematical tools used in previous sections, we obtain, $\boldsymbol{E}_{1}(x)$, hence the energy absorbed in this case:

$$
S_{\text {inhom }}=\frac{F_{05} \omega^{\prime \prime}}{2 \pi} \int\left(1-\frac{\omega_{p}^{2}}{\omega^{2}}\right)^{-1} \mathrm{~d} x
$$

Figure 8 shows that the sharp increase in the generated electric field due to $\frac{\partial v_{0 b}}{\partial x} \neq 0$-after a critical distance in the plasma-compared to the case $\frac{\partial v_{0 b}}{\partial x}=0$. This positively affects the plasma heating, and creates a type of plasma acceleration. 


\section{Conclusions and Final Remarks}

The Electron Beam Heating of Magneto-Active Motional Dusty Plasma is investigated.

Generally speaking, nonlinear process, dust, plasma motion, and beam velocity inhomogeneity, are found to play a crucial role via power absorbed by the beam and the generated electric field in the system.

It is shown that strong magnetic field generates an intense electric field compared to zero, and weak magnetic fields (Figure 1). In turn, an applied external static magnetic field leads to enhanced power absorption from the electron beam, and accordingly to plasma heating in beam-plasma system.

The energy absorbed from the beam in clean $(S)$ and dusty $\left(S_{d}\right)$ plasma (Figure 2), shows that dust causes a loss in the power energy absorbed in the plasma.

Considering nonlinear interaction, ratio (30) shows that dust leads to less energy absorption by the plasma as in the linear regime.

Figure 3 and Figure 4, shows the generated electric field under the effect of dusty and non-dusty plasmas in linear and nonlinear stages. In this regime amplification of electric field in dusty plasma is less compared to clean plasma. However, in clean plasms, the nonlinear effects associated with the generation of second harmonics, plays an important role in the process of energy transfer from the beam to the plasma as

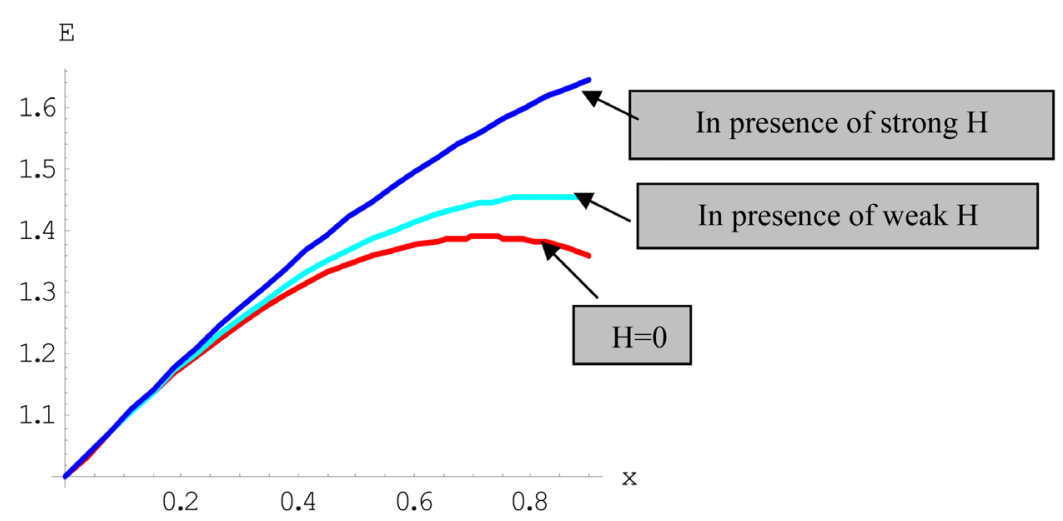

Figure 1. Generated electric field under the effect of zero, weak and strong magnetic fields.

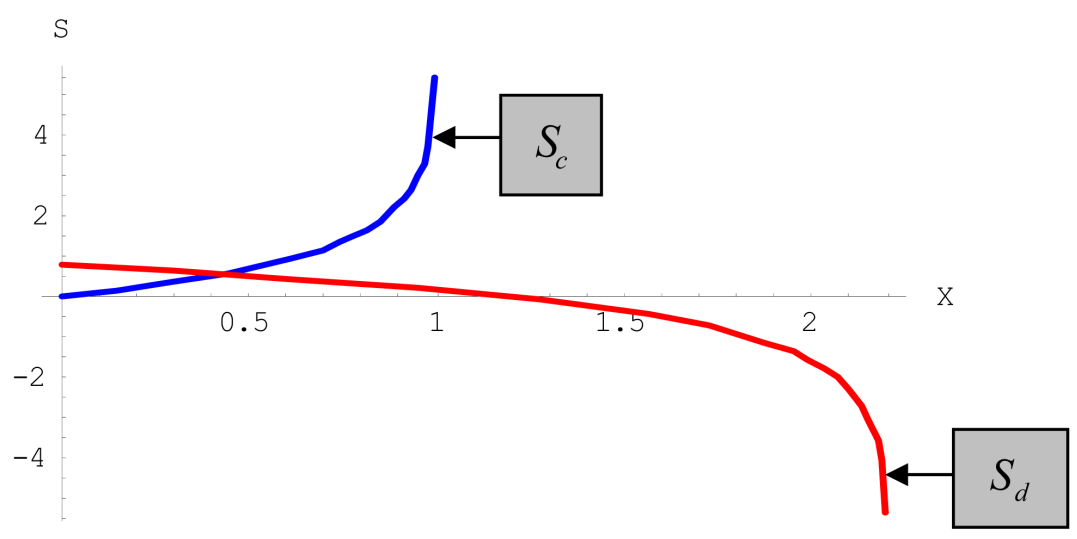

Figure 2. Energy absorbed from the beam in clean $(S)$ and dusty $\left(S_{d}\right)$ plasmas. 


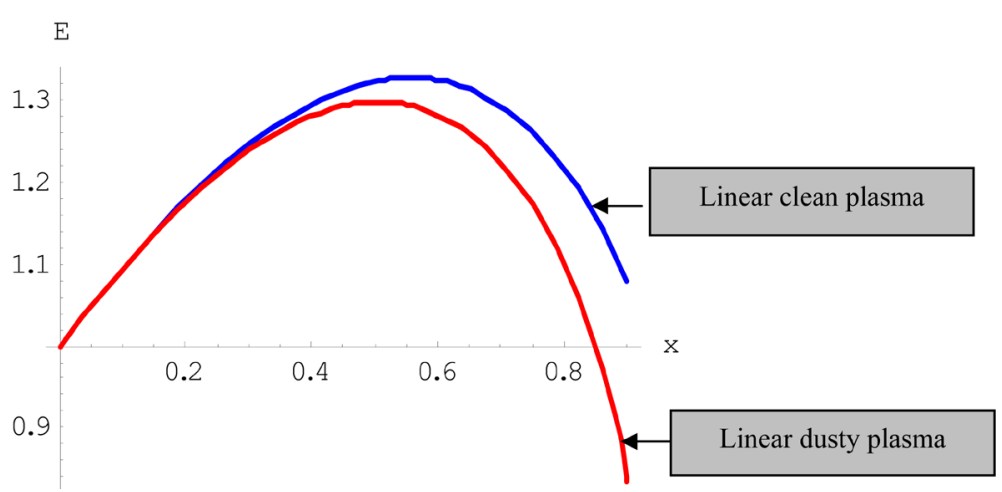

Figure 3. Generated electric field under the effect of dusty and clean linear plasma.

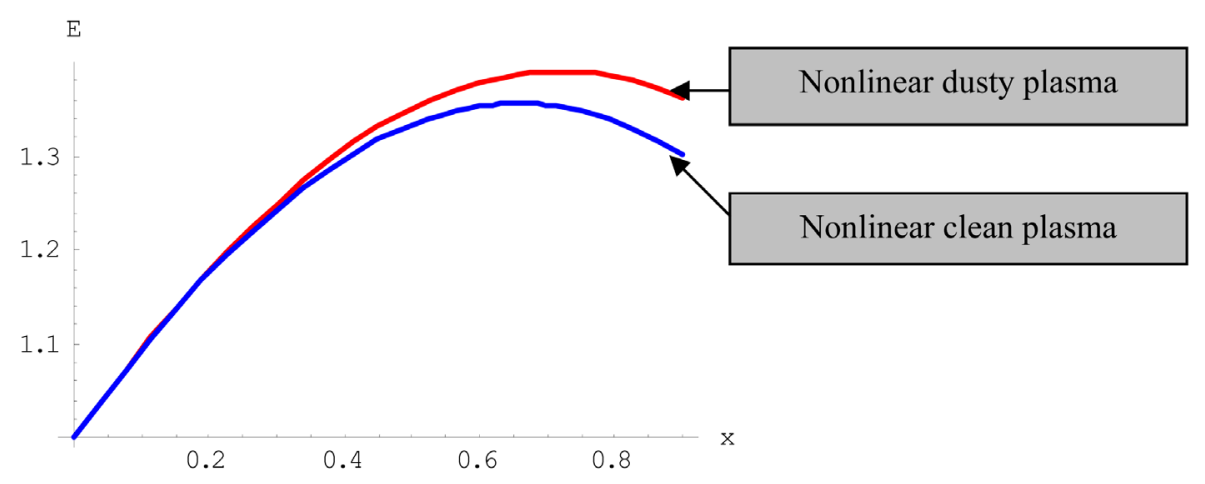

Figure 4. Generated electric field under the effect of dusty and clean nonlinear plasma.

compared with linear stage. This is due to the fact that the electric field intensity at double harmonics is stronger than that of the basic frequency (Figure 5 shows electric field amplification in nonlinear regime).

As application, the generation of second harmonics through a nonlinear mechanism driven by bunching at the fundamental has sparked interest as a path toward enhancing and extending the usefulness of an x-ray free-electron laser (FEL) facility [14]. Currently, high-order harmonic generation (HHG) is considered as one of the more efficient technique for producing coherent short-wavelength radiation in a broad spectral range [16] [24] [25].

In the motional plasma, the interaction between the electron beam and plasma will occur and induce electrons to exchange energy with plasma waves. The self-magnetic field of the motional electrons is a key parameter of this interaction.

Ratio (37) shows that, the existence of plasma motion leads to less energy absorption of the beam, as indicated in Figure 6. The reduction of the generated electric field under the effect of motional plasma compared to non-motional plasma, is shown in Figure 7.

An interesting result is shown in Figure 8, the sharp increase in the generated electric field due to beam velocity inhomogeneity $\frac{\partial v_{0 b}}{\partial x} \neq 0$-after a critical distance in the plasm compared to the case $\frac{\partial v_{0 b}}{\partial x}=0$. This enhanced power absorption of the electron 


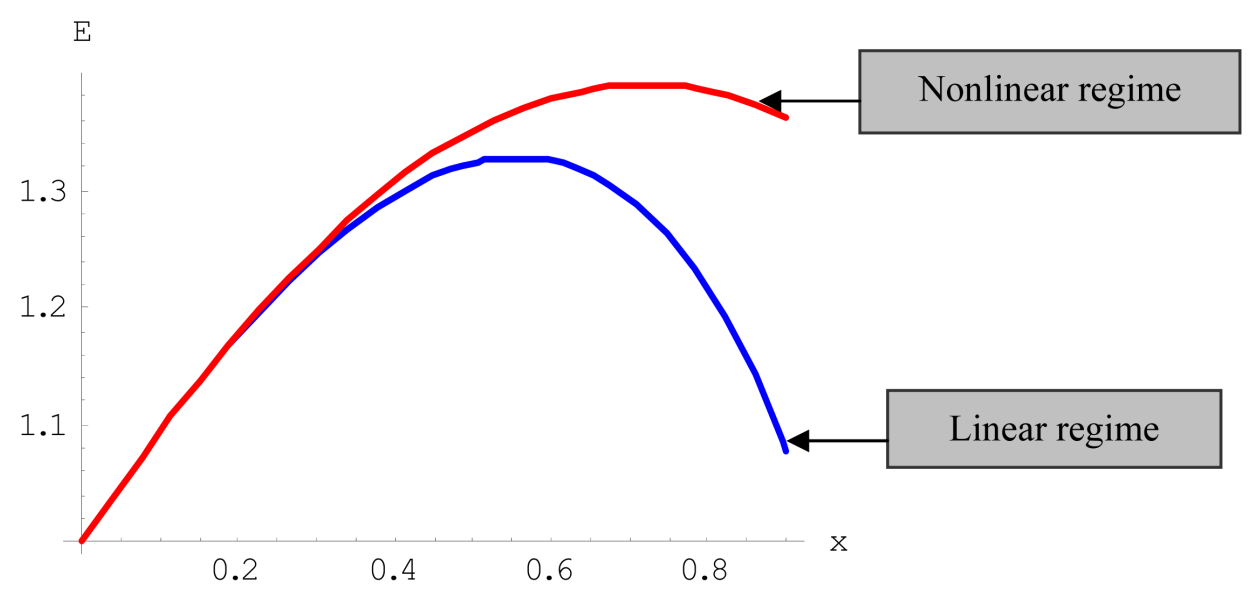

Figure 5. Electric field amplification in the nonlinear regime.

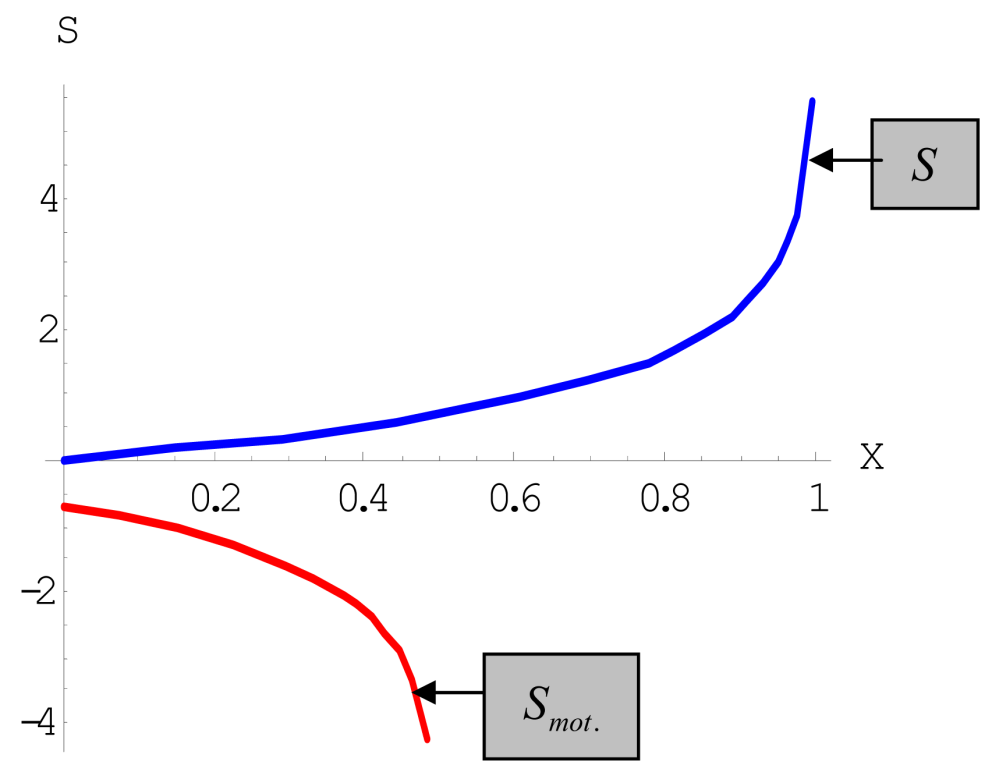

Figure 6. Energy absorbed from the beam in non-motional $(\mathrm{S})$ and motional $\left(\mathrm{S}_{\text {mot. }}\right)$ plasmas.

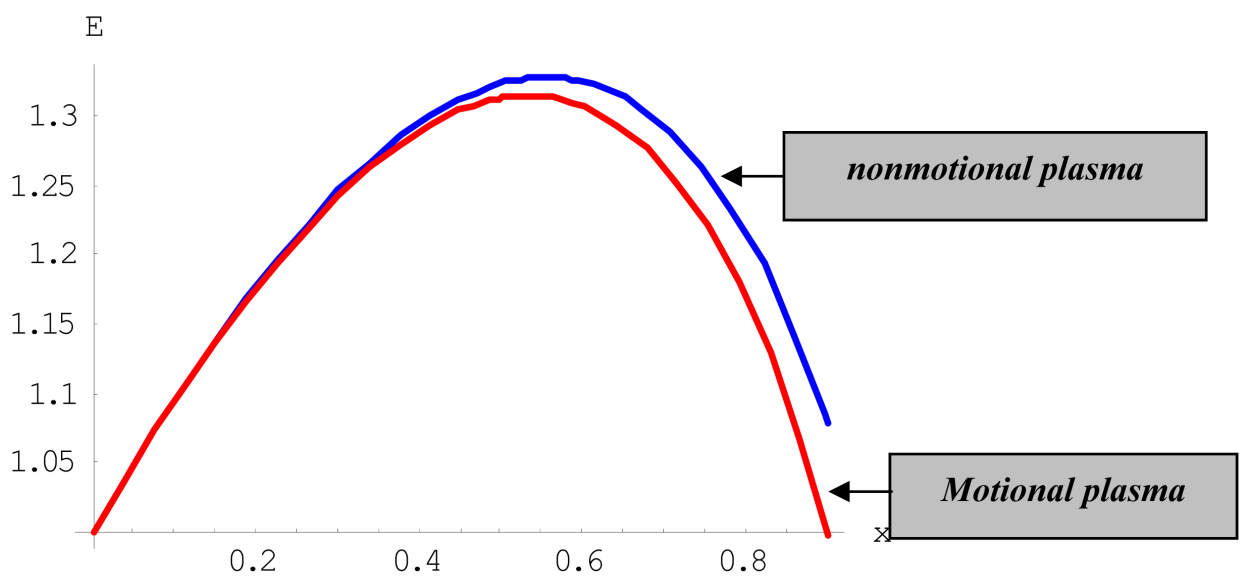

Figure 7. Generated electric field under the effect of motional and nonmotional plasma. 


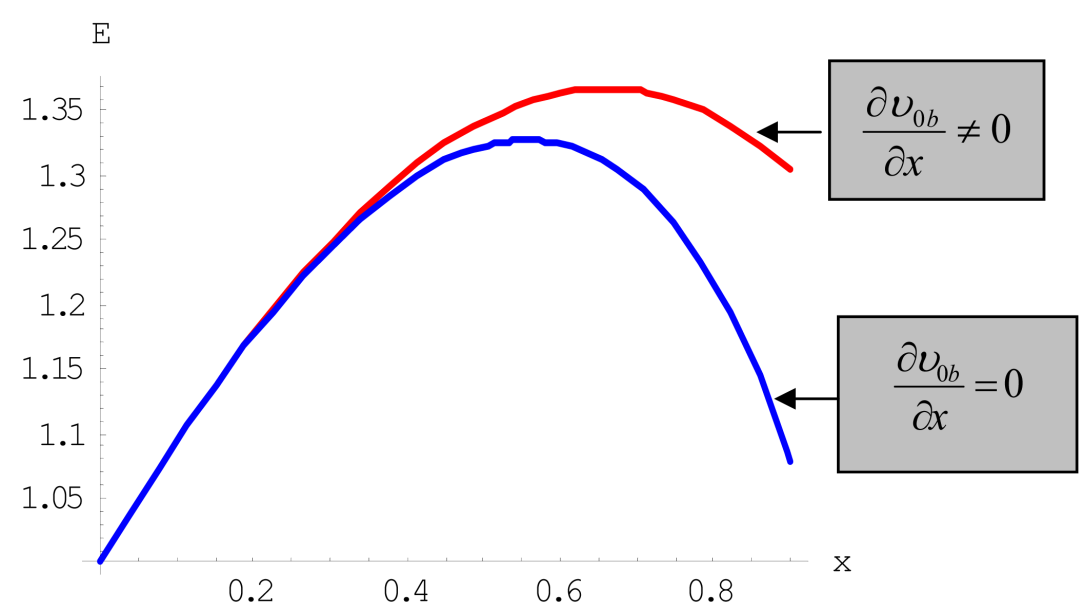

Figure 8. Generated electric field under the effect of inhomogeneous and homogeneous beam velocity.

beam, and positively affects the plasma heating, and may create a type of plasma acceleration.

In due course, we are going to investigate the effects of an inhomogeneous relativistic electron beam (REB) interaction with dusty, motional inhomogeneous plasma placed in an oscillating magnetic field.

\section{References}

[1] Khalil, Sh.M. and Al-Enazi, M.M. (2009) Advanced Studies in Theoretical Physics, 3, 369. http://www.m-hikari.com/astp/astp2009/astp9-12-2009/index.html

[2] Khalil, Sh.M. and Al-Enazi, M.M. (2010) Journal of Modern Physics, 2, 79. http://dx.doi.org/10.4236/jmp.2011.22013

[3] Karlický, M. (2009) The Astrophysical Journal, 690, 189. http://iopscience.iop.org/article/10.1088/0004-637X/690/1/189/meta

[4] Kartashov, N., Kuzelev, M.V. and Rukhadze, A.A. (2009) Plasma Physics Reports, 35, 169. http://dx.doi.org/10.1134/S1063780X09020093

[5] El-Sharif, R.N. and El-Shorbagy, Kh.H. (2013) Physics of Wave Phenomena, 21, 222. http://dx.doi.org/10.3103/S1541308X13030084

[6] Llor Aisa, E., et al. (2015) Physics of Plasmas, 22, Article ID: 102704. http://dx.doi.org/10.1063/1.4933119

[7] Johzaki, T., et al. (2015) Nuclear Fusion, 55, Article ID: 053022. http://dx.doi.org/10.1088/0029-5515/55/5/053022

[8] Lobaev, M.A., et al. (2016) Radiophysics and Quantum Electronics, 58, 816. http://dx.doi.org/10.1007/s11141-016-9656-Z

[9] Voronov, S.L., et al. (2001) Physics Review Letter, 87, Article ID: 133902. http://dx.doi.org/10.1103/PhysRevLett.87.133902

[10] Dahiya, D., et al. (2007) Physics of Plasmas, 14, Article ID: 123104. http://dx.doi.org/10.1063/1.2817088

[11] Gibbon, P. (1997) IEEE Journal of Quantum Electronics, 33, 1915. http://dx.doi.org/10.1109/3.641306 
[12] Jarque, E.C. and Plaja, L. (1998) Physics Review, E, 58, 7864.

http://dx.doi.org/10.1103/PhysRevE.58.7864

[13] Lichters, R., et al. (1996) Physics of Plasmas, 3, 3425. http://dx.doi.org/10.1063/1.871619

[14] Biedron, S.G., et al. (2002) Nuclear Instruments and Methods in Physics Research A, 483, 1. http://dx.doi.org/10.1016/S0168-9002(02)00292-9

[15] Biedron, S.G., et al. (2002) Physical Review-Special Topics-Accelerators and Beams, 5, Article ID: 030701. http://dx.doi.org/10.1103/PhysRevSTAB.5.030701

[16] Corkum, P.B. and Krausz, F. (2007) Nature Physics, 3, 381. http://dx.doi.org/10.1038/nphys620

[17] Bilikmen, S. and Nazih, M.R. (1993) Physica Scripta, 47, 204. http://iopscience.iop.org/article/10.1088/0031-8949/47/2/013

[18] Gupta, M.R., Sarkar, S., Khan, M. and Ghosh, S. (2003) Pramana Journal of Phys., Indian, 61, 1197. http://link.springer.com/article/10.1007/BF02704416

[19] Vranjes, J. and Tanaka, M.Y. (2002) Physical Review E, 66, Article ID: 037401. http://dx.doi.org/10.1103/PhysRevE.66.037401

[20] Rao, N.N. (1998) Physica Scripta, T75, 1506. http://iopscience.iop.org/article/10.1238/Physica.Topical.075a00179/pdf

[21] Ricci, P., et al. (2001) Physics of Plasmas, 8, 769. https://www.deepdyve.com/lp/american-institute-of-physics/plasma-kinetics-in-dusty-plas mas-IMFSzIh2Rv

[22] Johzahi, T., et al. (2003) Fusion Science and Technology, 43, 428. http://www.ans.org/store/article-288/

[23] Korenev, S. (2001) 19th IEEE Particle Accelerator Conference, Chicago, IL, 18-22 June 2001, 2332. http://dx.doi.org/10.1109/pac.2001.987369

[24] ozmus, W., et al. (2005) 32nd EPS Conference on Plasma Physics, Tarragona, 27 June-1 July 2005, 29C, O-1.006 http://citeseerx.ist.psu.edu/viewdoc/summary?doi=10.1.1.493.4476

[25] Dromey, B., et al. (2006) Nature Physics, 2, 456. http://dx.doi.org/10.1038/nphys338

\section{Submit or recommend next manuscript to SCIRP and we will provide best service for you:}

Accepting pre-submission inquiries through Email, Facebook, LinkedIn, Twitter, etc. A wide selection of journals (inclusive of 9 subjects, more than 200 journals)

Providing 24-hour high-quality service

User-friendly online submission system

Fair and swift peer-review system

Efficient typesetting and proofreading procedure

Display of the result of downloads and visits, as well as the number of cited articles Maximum dissemination of your research work

Submit your manuscript at: http://papersubmission.scirp.org/

Or contact jmp@scirp.org 